\title{
ULTRAVIOLET AND VIOLET RECEPTORS EXPRESS IDENTICAL mRNA ENCODING AN ULTRAVIOLET-ABSORBING OPSIN: IDENTIFICATION AND HISTOLOGICAL LOCALIZATION OF TWO mRNAS ENCODING SHORT-WAVELENGTH-ABSORBING OPSINS IN THE RETINA OF THE BUTTERFLY PAPILIO XUTHUS
}

\author{
JUNKO KITAMOTO ${ }^{1}$, KOICHI OZAKI ${ }^{2}$ AND KENTARO ARIKAWA ${ }^{1,3, *}$ \\ ${ }^{1}$ Graduate School of Integrated Science, Yokohama City University, 22-2 Seto, Kanazawa-ku, Yokohama 236-0027, \\ Japan, ${ }^{2}$ Graduate School of Science, Osaka University, 1-1 Machikaneyama, Toyonaka 560-0043, Japan and \\ ${ }^{3}$ PRESTO, Japan Science and Technology Corporation, 4-1-8 Honcho, Kawaguchi 332-0012, Japan
}

*Author for correspondence (e-mail: arikawa@yokohama-cu.ac.jp)

Accepted 3 July; published on WWW 7 September 2000

\begin{abstract}
Summary
This paper describes the primary structures of two opsins of short-wavelength-absorbing visual pigments deduced from the mRNA sequences in the retina of the Japanese yellow swallowtail butterfly Papilio xuthus. A phylogenetic analysis of the amino acid sequences indicates that one of these visual pigments is of the ultravioletabsorbing type and that the other is of the blue-absorbing type. We identified the photoreceptor cells that express these mRNAs by histological in situ hybridization. The mRNA of the ultraviolet type is expressed in two distinct

photoreceptor types previously identified as ultraviolet and violet receptors, providing the first molecular biological evidence that different types of spectral receptor probably express a visual pigment with an identical amino acid sequence. The mRNA of the blue type is expressed exclusively in cells classified as blue receptors.

Key words: colour, vision, insect, compound eye, ommatidium, retina, photoreceptor, rhodopsin, fluorescence, butterfly, Papilio xuthus.
\end{abstract}

\section{Introduction}

The compound eye of the Japanese yellow swallowtail butterfly Papilio xuthus is composed of approximately 12000 ommatidia, each containing nine photoreceptor cells, R1-R9 (Fig. 1). Intracellular recordings demonstrated that these cells can be classified into at least five different types of spectral receptor, with wavelengths of maximal absorption in the ultraviolet, violet, blue, green or red wavelength regions (Arikawa et al., 1987). Of the four distal photoreceptor cells, $\mathrm{R} 1-\mathrm{R} 4$, which contribute their microvilli to the rhabdom in the distal two-thirds of the ommatidium, R1 and R2 are ultraviolet, violet or blue receptors; R3 and R4 are always green receptors (Bandai et al., 1992). The R5-R8 cells, which contribute their microvilli in the proximal one-third of the rhabdom, are either green or red receptors, and R9, the basal photoreceptor, which adds some microvilli to the rhabdom immediately distal to the basement membrane, is also either a green or a red receptor (Arikawa and Uchiyama, 1996).

The ommatidia of Papilio xuthus are clearly spectrally heterogeneous, so how are the ommatidia different, and how are the different ommatidia arranged in the compound eye? An attractive strategy for determining the cellular organization of ommatidia is to clone the mRNAs of the visual pigment opsins and to localize them in the retina by histological in situ hybridization. Accordingly, we conducted a molecular biological study that yielded the mRNAs encoding the opsins of three long-wavelength-absorbing visual pigments and localized their expression in the retina (Kitamoto et al., 1998).

In addition to these three pigment mRNAs, we have now identified mRNAs encoding opsins for an ultraviolet-absorbing and a blue-absorbing visual pigment in the Papilio xuthus retina. Combining fluorescence microscopy, electrophysiology and in situ hybridization, we found that both ultraviolet and violet receptors express the mRNA of the ultraviolet-absorbing opsin. This lends molecular biological support to our previous hypothesis that the abnormally narrow sensitivity spectrum of violet receptors results from an ultraviolet-absorbing visual pigment that is spectrally filtered by an ultraviolet-absorbing screening pigment located in the same ommatidium (Arikawa et al., 1999b).

\section{Materials and methods} Animals

The Japanese yellow swallowtail butterfly Papilio xuthus L. was reared in the laboratory. The larvae, hatched from eggs laid by females caught in Yokohama, Japan, were fed on fresh citrus leaves at $25^{\circ} \mathrm{C}$ under a $16 \mathrm{~h}: 8 \mathrm{~h}$ light:dark photoperiod. Adults were used within 5 days of emergence. 


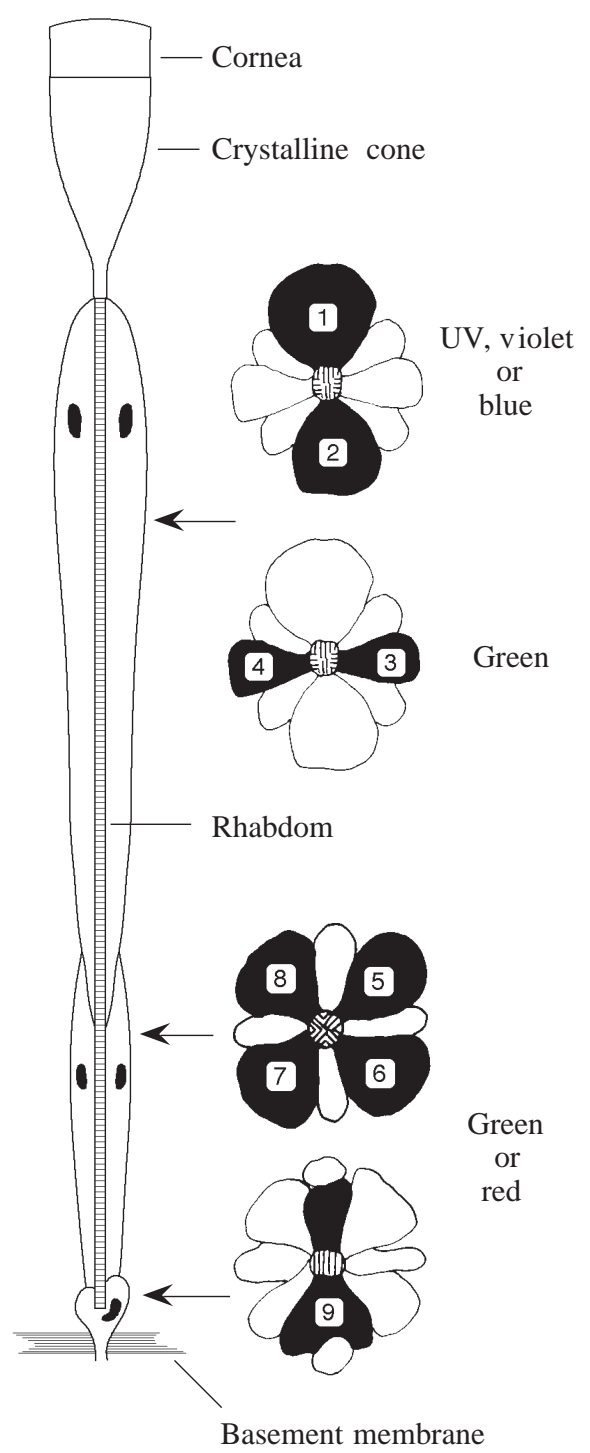

Fig. 1. Schematic drawing of a Papilio xuthus ommatidium, which is of the tiered type. $1-4$, distal photoreceptors R1-R4; 5-8, proximal photoreceptors R5-R8; 9, basal photoreceptor R9. UV, ultraviolet.

\section{Molecular cloning}

The method of molecular cloning of Papilio xuthus opsins was as described previously (Kitamoto et al., 1998). Briefly, retinal mRNA was first extracted using the QuickPrep Micro mRNA purification kit (Pharmacia) from eyes rapidly frozen in liquid nitrogen. To amplify the cDNAs of short-wavelengthabsorbing opsins using reverse transcription/polymerase chain reaction (RT-PCR), we designed a set of degenerate primers based on amino acid sequences conserved in short-wavelengthabsorbing opsins of the honeybee Apis mellifera (Townson et al., 1998), the locust Schistocerca gregaria (Towner et al., 1997), the hawkmoth Manduca sexta (Chase et al., 1997) and the tiger swallowtail Papilio glaucus (Briscoe, 1998) (see the legend of Fig. 2 for the sequences of the primers). Following RT-PCR using these primers, we identified two DNA fragments with opsin-like sequences. We labelled the fragments with ${ }^{32} \mathrm{P}$, and used them as probes to screen the Papilio retinal cDNA library. The isolated clones did not contain part of the coding region close to the $5^{\prime}$ end. This was later determined by $5^{\prime}$ rapid amplification of cDNA ends (RACE), and thus the full-length cDNAs were obtained (see Fig. 2).

\section{Comparison of amino acid sequences}

To compare the amino acid sequences deduced from the cloned cDNAs with opsins described in insects, the sequences were aligned using an alignment program (CLUSTAL W 1.6), and a phylogenetic analysis was then performed using the neighbour-joining method (PHYLIP 3.572), with octopus opsin as an outgroup.

\section{In situ hybridization}

The compound eyes of Papilio xuthus were fixed in $4 \%$ paraformaldehyde in $0.1 \mathrm{moll}^{-1}$ sodium phosphate buffer (PB; $\mathrm{pH} 7.2$ ) for $0.5-2 \mathrm{~h}$ at $25^{\circ} \mathrm{C}$ and preserved in $30 \%$ sucrose in $\mathrm{PB}$ at $4{ }^{\circ} \mathrm{C}$ overnight. The eyes were then embedded and frozen in Tissue-Tec OCT compound (Miles) and subsequently sectioned at $6-10 \mu \mathrm{m}$ thickness with a cryostat.

Probes for in situ hybridization were designed to hybridize to approximately 200 bases from the mRNAs at the non-coding region downstream of the $\mathrm{C}$ terminus. Corresponding cDNA regions were first subcloned into pGEM-3zf(+) vector, and digoxigenin (DIG)-labelled cRNAs were then generated using the DIG-RNA labelling kit (Boehringer Mannheim). The absence of cross-hybridization was confirmed by dot-blot analysis.

Serial sections from a single eye were mounted separately on several slides to label the sections with different probes. One of the slides from a single eye was always labelled with the probe Px-L1, which hybridizes one of the long-wavelengthabsorbing opsins we had previously identified, for precise identification of the dorsal and the ventral halves of the eye: Px-L1 is detected only in ventral regions (Kitamoto et al., 1998).

For labelling, sections were treated with hybridization solution containing $0.5 \mu \mathrm{g} \mathrm{ml}^{-1}$ of the cRNA probe at $65^{\circ} \mathrm{C}$ overnight. After a brief rinse, the sections were incubated in $50 \%$ formamide in $2 \times \mathrm{SSC}\left(1 \times \mathrm{SSC}\right.$ is $0.15 \mathrm{mmoll}^{-1} \mathrm{NaCl}$, $0.015 \mathrm{mmol}^{-1}$ sodium citrate) at $65^{\circ} \mathrm{C}$ for $2 \mathrm{~h}$, and then treated with RNAase $\left(10 \mu \mathrm{g} \mathrm{ml}^{-1}\right)$ at $37^{\circ} \mathrm{C}$ for $1 \mathrm{~h}$. The probes were visualized using anti-DIG immunocytochemistry.

\section{Fluorescence microscopy}

We previously found that some ommatidia of Papilio xuthus fluoresce under ultraviolet epi-illumination, both in vivo and in sections (Arikawa et al., 1999b; Arikawa and Stavenga, 1997). To correlate the ultraviolet-induced autofluorescence with the results of the in situ hybridization, we quickly video-recorded the fluorescence of the frozen sections, because the fluorescence easily bleaches under prolonged ultraviolet epiillumination. The frozen sections were then processed for in situ hybridization and further imaging. The video-recorded images were printed and then compared. 


\section{Results}

We identified two novel mRNAs encoding opsins in addition to the mRNAs of the opsins of the three longwavelength-absorbing visual pigments identified previously (Kitamoto et al., 1998). As we shall explain in detail below, it appeared that the encoded opsins are of the ultraviolet- and blue-absorbing types. We therefore term them Px-UV (Papilio xuthus-UV) and Px-B, respectively. For clarity, we here reterm the opsins of the long-wavelength-absorbing visual pigments PxRh1, PxRh2 and PxRh3 (Kitamoto et al., 1998) as Px-L1, Px-L2 and Px-L3 (for Papilio xuthus long-wavelengthabsorbing types $1-3$ ) respectively.

\section{Primary structures of two novel opsins}

The cDNAs of Px-UV and Px-B have single open reading frames of $1140 \mathrm{bp}$ and $1143 \mathrm{bp}$, encoding 380 and 381 amino acid residues, respectively (Fig. 2). Both Px-UV and Px-B have amino acid residues conserved in other opsins, such as lysine in the seventh helix for chromophore attachment (Wang et al., 1980) and cysteines for disulphide bond formation (Karnik et al., 1988). The amino acid residue in the third helix corresponding to the Schiff base counter-ion in vertebrate opsins was phenylalanine in Px-UV and tyrosine in Px-B.

Fig. 3 shows the phylogenetic relationships of the insect opsins identified to date, including Px-UV and Px-B. Px-UV falls into the group of the ultraviolet-absorbing types, whereas $\mathrm{Px}-\mathrm{B}$ is in the group of the blue-absorbing types.

\section{Histological in situ hybridization}

The photoreceptor cells expressing Px-UV and Px-B were identified by histological in situ hybridization on frozen sections of the eye. Histological in situ hybridization demonstrates only that the photoreceptor cells contain mRNAs, not Px-UV and Px-B themselves, although these mRNAs are likely to be translated into functional proteins.

Fig. 4A,C shows oblique sections labelled with the Px-UV and the Px-B probe, respectively. The labelling was found exclusively in the distal two-thirds (Fig. 4A) or the middle portion (Fig. 4C) of the photoreceptor layer. The distal photoreceptors bear their rhabdomeral microvilli in the distal two-thirds of the rhabdom (Arikawa and Uchiyama, 1996). The densely labelled portion corresponds to the region immediately proximal to their nuclei. At any rate, the proximal photoreceptors (R5-R8), which contribute their microvilli only to the proximal one-third of the rhabdom, are not labelled with these probes.

The other five sections shown in Fig. 4 were obtained from the same region of an eye: the white polygon indicates the corresponding area on each section. Fig. 4E is the ommatidial autofluorescence photographed immediately after the section was cut in the cryostat. Fig. 4B,D,F,G were labelled with probes that hybridize specifically to the mRNAs of Px-UV, PxB, Px-L2 and Px-L3, respectively.

In the distal tier, the mRNAs of Px-UV and Px-B were found only in R1 and R2 photoreceptors: the other photoreceptors, R3-R9, were not labelled. In Fig. 4B,D, there are three types of ommatidia in terms of the mRNA they contain in the R1 and $\mathrm{R} 2$ photoreceptors. Type 1 ommatidia have Px-UV mRNA in $\mathrm{R} 1$ and Px-B mRNA in R2 (right-hand red circle) or vice versa (left-hand red circle). Type 2 has Px-UV mRNA in both R1 and R2 (pink circle). This type corresponds to those that emit strong fluorescence under ultraviolet epi-illumination (Fig. 4E). The other ommatidia, which contain the Px-B mRNA in both R1 and R2, are type 3 (yellow circle). By comparing Fig. 4B and Fig. 4D, it is clear that all R1 and R2 photoreceptors are labelled with either one of these probes and

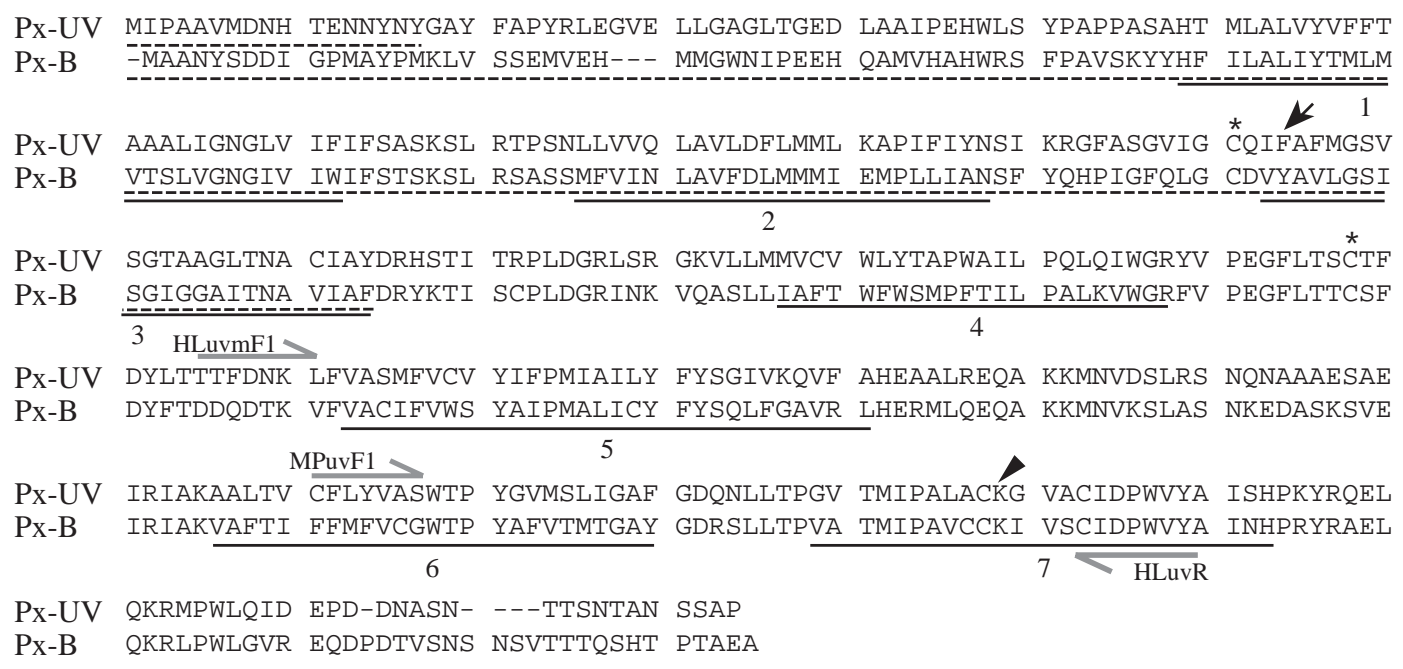

Fig. 2. Deduced amino acid sequences of opsins Px-UV and Px-B. Hatched half-arrows indicate regions corresponding to primer attachment: the primer sequences are GCGAATTCGAYGAYGARGAYACNAARGT (HLuvmF1), GCGAATTCTGYTTYYTNTTYGTNGC (MPuvF1) and CGGGATCCTANAYCCANGGRTCDATRCA (HLuvR). Broken underlining indicates the regions determined by 5' RACE. Large arrow, position corresponding to the Schiff base counter-ion in vertebrate opsins; arrowhead, lysine for chromophore attachment; asterisks, cysteines for disulphide bond formation. Data accession numbers for Px-UV and Px-B are AB028218 and AB028217, respectively. 


\section{J. Kitamoto, K. OzaKi AND K. ARIKaWA}

Fig. 3. Phylogenetic relationship of insect visual pigment opsins calculated using the neighbourjoining method with octopus opsin as the outgroup. Wavelengths indicate the wavelength of maximal absorption $\left(\lambda_{\max }\right)$ of the absorption spectrum of these visual pigments estimated by intracellular electrophysiology of animals in situ [Schistocerca gregaria, Bennett et al. (1967); Manduca sexta, White et al. (1983); Apis mellifera, Apis green, Menzel et al. (1986)] or by electroretinogram measurement of transgenic Drosophila melanogaster in which the corresponding opsins are expressed [Apis UV and Apis blue, Townson et al. (1998); Drosophila Rh1, Zuker et al. (1988); Drosophila Rh2, Feiler et al. (1988); Drosophila Rh3 and Rh4, Feiler et al. (1992); Drosophila Rh5 and Rh6, Salcedo et al. (1999)]. Data accession numbers: Apis UV, AF004169; Apis blue, AF004168; Apis green, U26026; Camponotus, U32502; Cataglyphis, U32501; D. melanogaster Rh1, A90864; D. melanogaster Rh2, A24058; D. melanogaster Rh3, Y00043; D. melanogaster Rh4, M17730; D. melanogaster Rh5, U80667; D. melanogaster Rh6, Z86118; Manduca 1, L78080; Manduca 2, L78080; Manduca 3, AD001674; Papilio xuthus Px-L1 (PxRh1), AB007423; Papilio xuthus Px-L2 (PxRh2), AB007424; Papilio xuthus Px-L3 (PxRh3), AB007425; Schistocerca Lo1, X80071; Schistocerca Lo2, X80072.

also that no R1 and R2 photoreceptors are double-labelled with them.

Fig. 4F,G are adjacent sections through the proximal tier. These sections were labelled with probes detecting Px-L2 (Fig. 4F) and Px-L3 (Fig. 4G) mRNAs, which have been shown to be expressed in the green and red receptors (Kitamoto et al., 1998). In Fig. 4F, the labelling density of the R5-R8 proximal photoreceptors is evidently variable between ommatidia: no labelling, weak labelling and strong labelling all occurred (Arikawa, 1999; Kitamoto et al., 1998). Apparently, these variations correspond to three ommatidial types; i.e. no labelling for type 1; weak labelling for type 2 and strong labelling for type 3 . The R5-R8 photoreceptors of type 2 ommatidia co-express Px-L3 mRNA (Fig. 4G).

Fig. $4 \mathrm{H}$ shows the organization of three types of ommatidia in the area encircled by the polygon. Of the 32 ommatidia in this particular tissue, 18 are of type $1(56.3 \%)$, six are of type $2(18.7 \%)$ and eight are of type $3(25.0 \%)$.

\section{Discussion \\ $P x-U V$ and $P x-B$ opsins}

In this paper, we describe two novel mRNAs encoding visual pigment opsins identified in the compound eye of Papilio xuthus. Histological in situ hybridization showed that these opsin mRNAs are expressed exclusively in the R1 or R2 photoreceptors in the distal tier of the ommatidia. Previously, we found that R1 and R2 are ultraviolet, violet or blue receptors (Bandai et al., 1992) and, hence, we conclude that the opsins are short-wavelength-absorbing visual pigments.

We have compared the amino acid sequences of the novel opsins deduced from the cDNA sequences with those of other insect opsins (Fig. 3). The phylogenetic analysis indicates that one of the new opsins is a member of the group including Manduca 2 (Chase et al., 1997), Apis UV (Townson et al., 1998), Drosophila Rh3 (Zuker et al., 1987) and Drosophila Rh4 (Montell et al., 1987), all presumed to be of the ultravioletabsorbing type. The other appears in the group of blueabsorbing opsins, including Manduca 3 (Chase et al., 1997), Apis blue (Townson et al., 1998) and Drosophila Rh5 (Chou et al., 1996). Together with our in situ hybridization results, discussed below, this indicates that these novel opsins are of the ultraviolet- and blue-absorbing class, respectively. We therefore termed these opsins as Px-UV and Px-B. Convincing proof of the spectral absorbance characteristics of the visual pigments could be provided by expression experiments, but these have not yet been completed.

\section{Violet and ultraviolet receptors share Px-UV mRNA}

The violet receptor in the retina of Papilio xuthus exhibits an abnormally narrow sensitivity spectrum (Arikawa et al., 1987). The physiological basis of the narrow sensitivity spectrum has only recently been resolved. In the ventral half of the eye, some ommatidia emit strong, whitish fluorescence 
Fig. 4. In situ hybridization in serial sections of the same region of the eye (not A and C). Identical ommatidia are marked by coloured symbols. (A) Oblique section labelled with the Px-UV probe. The distal tier (d) is labelled, whereas the proximal tier (p) is free of labelling. BM, basement membrane. (B) Transverse section through the distal tier labelled with the Px-UV probe. (C) Oblique section labelled with the Px-B probe. Only the distal tier is labelled. (D) Transverse section through the distal tier labelled with the Px-B probe. (E) Transverse section through the most distal part of the retina; ultraviolet-induced autofluorescence. (F) Transverse section of the proximal tier labelled with the Px-L2 probe. (G) Transverse section of the proximal tier labelled with the Px-L3 probe. (H) Three types of ommatidia are identified: red, type 1 ; pink, type 2 ; yellow, type 3 . These colours correspond to the transmission of each ommatidial type in eyeslice preparations (see Arikawa and Stavenga, 1997). Arrows indicate the ommatidia indicated by coloured circles in B and D-G. See also Tables 1 and 2. $D$, dorsal; $V$, ventral; Scale bars, $100 \mu \mathrm{m}$ (A,C); $60 \mu \mathrm{m}$ (B,D-G).
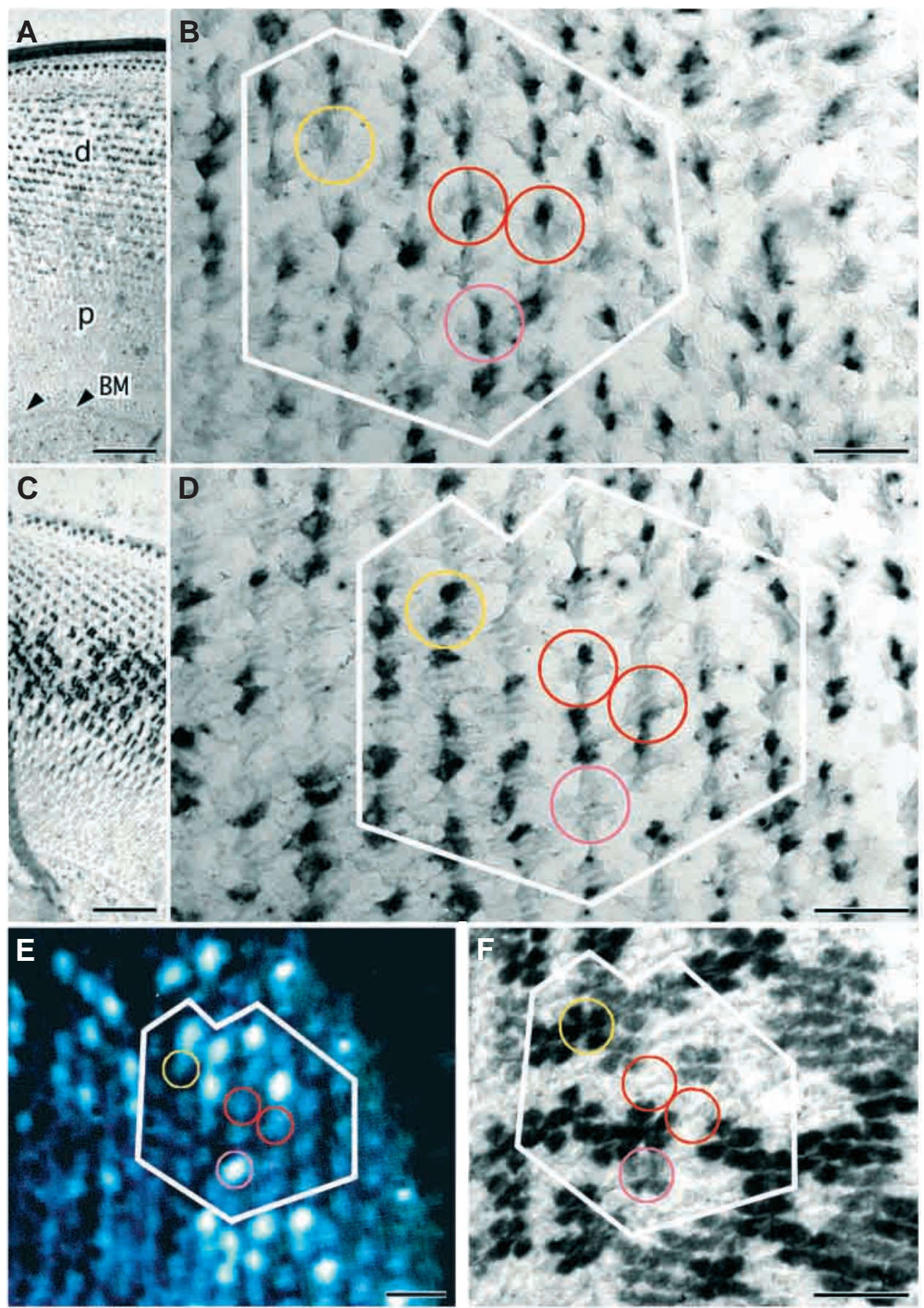

H

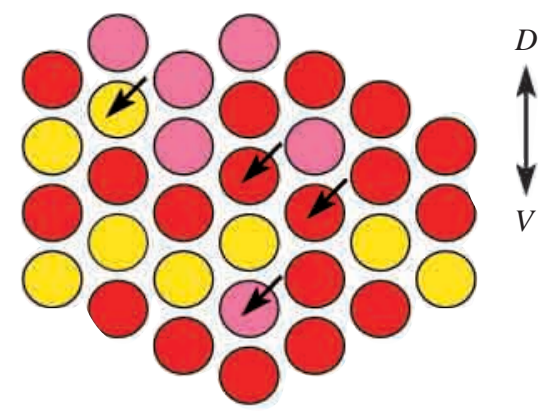

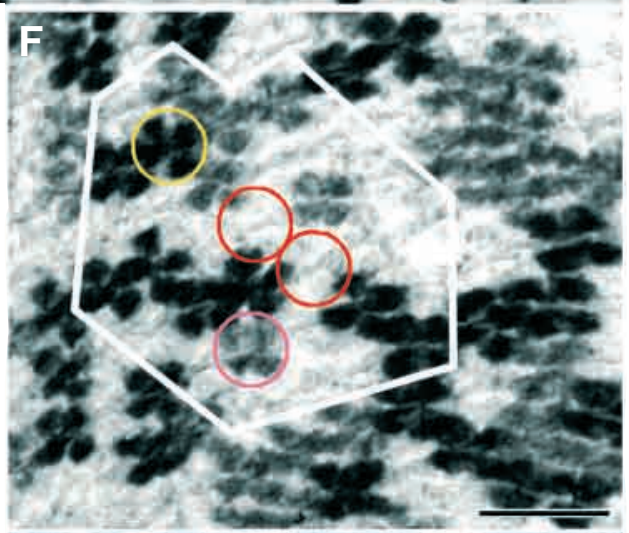

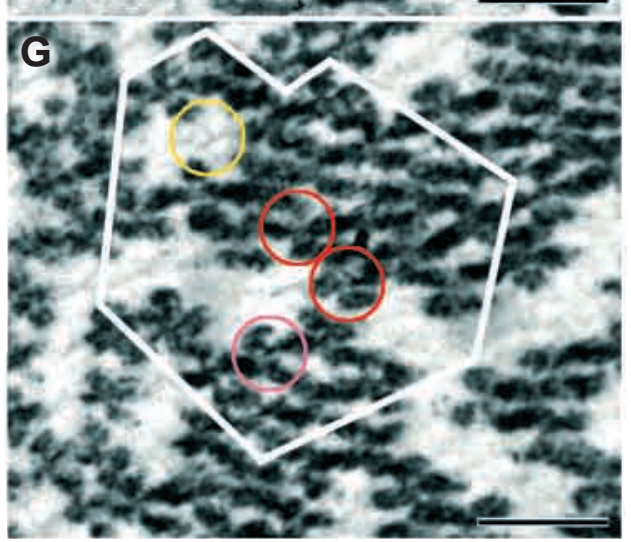




\section{J. Kitamoto, K. OzaKi And K. ARIKawa}

when illuminated by ultraviolet light (Arikawa and Stavenga, 1997). The fluorescing material, probably 3-hydroxyretinol (Arikawa et al., 1999b), is concentrated in the distal one-fifth of the ommatidia (Fig. 4E; see Fig. 2 in Arikawa et al., 1999b). This localization suggested a specific function of the 3hydroxyretinol; namely, that it acts as an ultraviolet-absorbing spectral filter for the photoreceptors underneath. We therefore constructed an optical model taking into account the detailed anatomy of Papilio xuthus ommatidia. When the R1 and R2 photoreceptors in the fluorescing ommatidia contain an ultraviolet-absorbing visual pigment, the model predicts that the sensitivity spectrum of the photoreceptors will become distinctly narrower than the absorption spectrum of the ultraviolet visual pigment and, furthermore, that the peak will be shifted to the violet, just as is found for the sensitivity spectrum of the violet receptor. The appropriateness of the ultraviolet-filter hypothesis was strengthened by further electrophysiological experiments: violet receptors were always found in the fluorescing ommatidia, whereas ultraviolet receptors were found only in the non-fluorescing ommatidia (Arikawa et al., 1999b).

In the simplest version of the above hypothesis, we assumed that both the violet and the ultraviolet receptors have the same ultraviolet-absorbing visual pigment. Almost to our surprise, the present in situ hybridization results strongly suggest that this is indeed the case. One could argue that the Px-UV probe might have detected multiple mRNAs encoding ultraviolet visual pigments with similar sequences each separately expressed in the ultraviolet receptors and the violet receptors. However, our probe was designed to hybridize to the noncoding region downstream from the $\mathrm{C}$ terminus, so that there is very little likelihood that the Px-UV probe hybridizes to two different mRNAs in this position. As is clearly demonstrated in Fig. 4, the fluorescing ommatidia contain Px-UV in both R1 and R2.

Px-UV is also expressed in the R1 or R2 photoreceptors in some other, non-fluorescing ommatidia. The Px-UV-
Table 1. Frequency of three types of ommatidia in the ventral and dorsal regions of the compound eye of Papilio xuthus

\begin{tabular}{lcc}
\hline Type (opsins in R1/R2) & $\begin{array}{c}\text { Ventral } \\
(\%)\end{array}$ & $\begin{array}{c}\text { Dorsal } \\
(\%)\end{array}$ \\
\hline Type 1 (Px-UV/Px-B) & $41.4(1535)$ & $40.7(177)$ \\
Type 2 (Px-UV/Px-UV) & $31.9(1182)$ & $8.7(38)$ \\
Type 3 (PX-B/Px-B) & $26.7(988)$ & $50.6(220)$ \\
Total & $100(3705)$ & $100(435)$
\end{tabular}

Numbers of ommatidia observed are given in parentheses.

containing R1 and R2 photoreceptors then behave as ultraviolet receptors.

The above discussion has focused only to the ventral half of the eye, where the type 2 ommatidia are always of the fluorescing type. Ommatidial fluorescence is obscured in the dorsal half of the eye, probably because of corneal fluorescence (Arikawa et al., 1999b). Although making only minor contribution, type 2 ommatidia are present in the dorsal half of the eye (Table 1). The R1 and R2 photoreceptors could therefore be ultraviolet receptors, but this has yet to be demonstrated electrophysiologically.

\section{Blue receptors}

The R1 and R2 photoreceptors are ultraviolet, violet or blue receptors (Bandai et al., 1992). These photoreceptors are labelled with either the Px-UV or the Px-B probe. As discussed above, the ultraviolet and violet receptors contain Px-UV mRNA. Hence, the R1 and R2 photoreceptors labelled with the Px-B probe must be blue receptors.

\section{An identical visual pigment in different spectral receptors}

The present results suggest that a visual pigment of identical amino acid sequence exists in the different spectral receptors that were identified electrophysiologically. The existence of identical visual pigments in different spectral receptors has

Table 2. Three types of ommatidia in the compound eye of Papilio xuthus

\begin{tabular}{|c|c|c|c|c|c|c|}
\hline \multirow[t]{2}{*}{$\begin{array}{l}\text { Fluorescence: } \\
\text { Pigmentation: }\end{array}$} & \multicolumn{2}{|c|}{$\begin{array}{c}\text { Type } 1 \\
\text { No } \\
\text { Red }\end{array}$} & \multicolumn{2}{|c|}{$\begin{array}{c}\text { Type } 2 \\
\text { Yes } \\
\text { Red }\end{array}$} & \multicolumn{2}{|c|}{$\begin{array}{c}\text { Type } 3 \\
\text { No } \\
\text { Yellow }\end{array}$} \\
\hline & $S(\lambda)$ & Opsin & $S(\lambda)$ & Opsin & $S(\lambda)$ & Opsin \\
\hline R1 & $\mathrm{UV}^{*}$ & Px-UV* & $\mathrm{V}$ & Px-UV & B & Px-B \\
\hline $\mathrm{R} 2$ & $\mathrm{~B}^{*}$ & Px-B* & $\mathrm{V}$ & Px-UV & B & Px-B \\
\hline R3-R4 & DG & $\mathrm{Px}-\mathrm{L} 1+\mathrm{L} 2$ & SG & $\mathrm{Px}-\mathrm{L} 1+\mathrm{L} 2$ & DG & $\mathrm{Px}-\mathrm{L} 1+\mathrm{L} 2$ \\
\hline
\end{tabular}

Spectral sensitivity, $S(\lambda)$, and the visual pigment opsins expressed in photoreceptors in the ventral half of the eye are shown.

UV, ultraviolet; V, violet; B, blue; DG, double-peaked green; SG, single-peaked green; R, red; A, abnormally broad.

Photoreceptors R3 and R4 of all ommatidia co-express two visual pigments.

For details of $S(\lambda)$ of UV, V, DG and SG pigments, see Bandai et al. (1992) and Arikawa et al. (1999b).

For $S(\lambda)$ of R and A pigments, see Arikawa et al. (1999c) and Arikawa et al. (1999a), respectively.

For visual pigments, see Kitamoto et al. (1998).

For Type 1, the spectral type of R1 and R2 may be interchanged (asterisks). 
been suggested previously in birds. In chickens, for example, the visual pigment absorbing at $571 \mathrm{~nm}$ appeared to be contained both in the principal member of the double cone containing greenish oil droplet and in the single cone containing reddish oil droplet. Because of the difference in the colour of the oil droplets, which act as spectral filters, the predicted absorption spectra for the outer segments of the double cone principal member and the single cone are distinctly different: the spectrum of the single cone is narrow and the peak is shifted to $600 \mathrm{~nm}$ (Bowmaker and Knowles, 1977; Okano et al., 1995).

However, in birds, electrophysiological measurements of the sensitivity spectrum of each photoreceptor are lacking. In this regard, the present case of Papilio xuthus fills the gap, although the presence of the identical visual pigment molecule itself in the ultraviolet and violet receptors has yet to be demonstrated by, for example, immunocytochemistry using specific antibodies.

\section{Cellular organization of three types of Papilio xuthus ommatidium}

We have suggested that there are at least three types of spectrally distinct ommatidia in the eye of Papilio xuthus on the basis of the distribution of colour filters (Arikawa and Stavenga, 1997) and of the mRNAs encoding longwavelength-absorbing opsins (Kitamoto et al., 1998). Here, we again characterized three types of ommatidia on the basis of the mRNA encoding the short-wavelength-absorbing opsins they contain. Taking all the information together, we now have an almost complete picture of the cellular organization of the ommatidia of Papilio xuthus (Table 2).

We showed previously that both Px-L1 and Px-L2 mRNAs are expressed in green receptors, whereas Px-L3 mRNA is expressed in red receptors (Kitamoto et al., 1998). Moreover, an electrophysiological study revealed that green-sensitive receptors R5-R8 are always found in yellow-pigmented ommatidia and that red-sensitive receptors R5-R8 are found exclusively in red-pigmented ommatidia (Arikawa et al., 1999c). Type 1 ommatidia with Px-L3 mRNA in R5-R8 photoreceptors (i.e. red receptors) must have red pigmentation. Similarly, type 3 ommatidia whose R5-R8 photoreceptors contain Px-L2 mRNA are yellow-pigmented. R3 and R4 photoreceptors in type 1 and type 3 ommatidia are normal green receptors with a secondary peak in the ultraviolet wavelength region (double-peaked green), which doubleexpress Px-L1 and Px-L2 mRNAs.

We also showed that some R5-R8 photoreceptors are double-labelled by the Px-L2 and Px-L3 probes (Fig. 4E,G; see also Kitamoto et al., 1998). Clearly, the ommatidia containing the double-labelled R5-R8 photoreceptors are of type 2 (Fig. 4). Another characteristic of type 2 ommatidia is ultraviolet-induced fluorescence (Arikawa et al., 1999b). The fluorescing ommatidia belong to a subset of red-pigmented ommatidia (Arikawa and Stavenga, 1997). Interestingly, the sensitivity spectra of the R5-R8 photoreceptors in the fluorescing ommatidia is abnormally broad (i.e. the half- bandwidth is approximately $270 \mathrm{~nm}$ ), suggesting different absorption spectra for the two expressed visual pigments. The anomalous cells are called A cells (Arikawa et al., 1999a).

The properties of R9 photoreceptors need further elucidation.

We thank D. G. Stavenga for critical reading of the manuscript. This work was supported by grants from the Japan Science Society and the Circle for the Promotion of Science and Engineering to J.K. and from the Ministry of Education, Science, and Culture of Japan to K.A.

\section{References}

Arikawa, K. (1999). Color vision. In Atlas of Sensory Receptors of Arthropods - Dynamic Morphology in Relation to Function (ed. E. Eguchi and Y. Tominaga), pp. 23-32. Tokyo: Springer Verlag.

Arikawa, K., Inokuma, K. and Eguchi, E. (1987). Pentachromatic visual system in a butterfly. Naturwissenschaften 74, 297-298.

Arikawa, K., Mizuno, S., Kinoshita, M. and Stavenga, D. G. (1999a). Two visual pigments simultaneously expressed in a subset of proximal photoreceptors of the butterfly, Papilio xuthus, cause an abnormally-broad spectral sensitivity. In Goettingen Neurobiology Report 1999 (ed. N. Elsner and U. Eisel), p. 409. Stuttgart, New York: Georg Thieme Verlag.

Arikawa, K., Mizuno, S., Scholten, D. G. W., Kinoshita, M., Seki, T., Kitamoto, J. and Stavenga, D. G. (1999b). An ultraviolet absorbing pigment causes a narrow-band violet receptor and a single-peaked green receptor in the eye of the butterfly Papilio. Vision Res 39, 1-8.

Arikawa, K., Scholten, D. G. S., Kinoshita, M. and Stavenga, D. G. (1999c). Tuning of photoreceptor spectral sensitivities by red and yellow pigments in the butterfly Papilio xuthus. Zool. Sci. 16, 17-24.

Arikawa, K. and Stavenga, D. G. (1997). Random array of colour filters in the eyes of butterflies. J. Exp. Biol. 200, 2501-2506.

Arikawa, K. and Uchiyama, H. (1996). Red receptors dominate the proximal tier of the retina in the butterfly Papilio xuthus. J. Comp. Physiol. A 178, 55-61.

Bandai, K., Arikawa, K. and Eguchi, E. (1992). Localization of spectral receptors in the ommatidium of butterfly compound eye determined by polarization sensitivity. J. Comp. Physiol. A 171, 289-297.

Bennett, R. R., Tunstall, J. and Horridge, G. A. (1967). Spectral sensitivity of single retinula cells in the locust. Z. Vergl. Physiol. 55, 195-206.

Bowmaker, J. K. and Knowles, A. (1977). The visual pigments and oil droplets of the chicken retina. Vision Res. 17, 755-764.

Briscoe, A. D. (1998). Molecular diversity of visual pigments in the butterfly Papilio glaucus. Naturwissenschaften 85, 33-35.

Chase, M. R., Bennett, R. R. and White, R. H. (1997). Three opsinencoding cDNAs from the compound eye of Manduca sexta. J. Exp. Biol. 200, 2469-2478.

Chou, W. H., Hall, K. J., Wilson, D. B., Wideman, C. L., Townson, S. M., Chadwell, L. V. and Britt, S. G. (1996). Identification of a novel Drosophila opsin reveals specific patterning of the R7 and R8 photoreceptor cells. Neuron 17, 1101-1115.

Feiler, R., Bjornson, R., Kirschfeld, K., Mismer, D., Rubin, G. M., Smith, D. P., Socolich, M. and Zuker, C. S. (1992). Ectopic 


\section{J. Kitamoto, K. OzaKi AND K. ARIKaWA}

expression of ultraviolet-rhodopsins in the blue photoreceptor cells of Drosophila - visual physiology and photochemistry of transgenic animals. J. Neurosci. 12, 3862-3868.

Feiler, R., Harris, W. A., Kirschfeld, K., Werhan, C. and Zuker, C. S. (1988). Targeted misexpression of a Drosophila opsin gene leads to altered visual function. Nature 333, 737-741.

Karnik, S. S., Sakmar, T. P., Chen, H.-B. and Khorana, H. G. (1988). Cysteine residues 110 and 187 are essential for the formation of correct structure in bovine rhodopsin. Proc. Natl. Acad. Sci. USA 85, 8459-8463.

Kitamoto, J., Sakamoto, K., Ozaki, K., Mishina, Y. and Arikawa, K. (1998). Two visual pigments in a single photoreceptor cell: identification and histological localization of three mRNAs encoding visual pigment opsins in the retina of the butterfly Papilio xuthus. J. Exp. Biol. 201, 1255-1261.

Menzel, R., Ventura, D. F., Hertel, H., de Souoza, J. M. and Greggers, U. (1986). Spectral sensitivity of photoreceptors in insect compound eyes: comparison of species and methods. $J$. Comp. Physiol. A 158, 165-177.

Montell, C., Jones, K., Zuker, C. and Rubin, G. (1987). A second opsin gene expressed in the ultraviolet-sensitive R7 photoreceptor cells of Drosophila melanogaster. J. Neurosci. 75, 1558-1566.

Okano, T., Fukada, Y. and Yoshizawa, T. (1995). Molecular basis for tetrachromatic color vision. Comp. Biochem. Physiol. 112B, 405-414.

Salcedo, E., Huber, A., Henrich, S., Chadwell, L. V., Chou, W. H., Paulsen, R. and Britt, S. G. (1999). Blue- and green-absorbing visual pigments of Drosophila: Ectopic expression and physiological characterization of the R8 photoreceptor cell-specific Rh5 and Rh6 rhodopsins. J. Neurosci. 19, 10716-10726.

Towner, P., Harris, P., Wolstenholme, A. J., Hill, C., Worm, K. and Gartner, W. (1997). Primary structure of locust opsins: A speculative model which may account for ultraviolet wavelength light detection. Vision Res. 37, 495-503.

Townson, S. M., Chang, B. S. W., Salcedo, E., Chadwell, L. V., Pierce, N. E. and Britt, S. G. (1998). Honeybee blue-and ultraviolet-sensitive opsins: Cloning, heterologous expression in Drosophila and physiological characterization. J. Neurosci. 18, 2412-2422.

Wang, J. K., McDowell, J. H. and Hargrave, P. A. (1980). Site of attachment of 11-cis-retinal in bovine rhodopsin. Biochemistry 19, 5111-5117.

White, R. H., Brown, P. K., Hurley, A. K. and Bennett, R. R. (1983). Rhodopsins, retinula cell ultrastructure and receptor potentials in the developing pupal eye of the moth Manduca sexta. J. Comp. Physiol. 150, 153-163.

Zuker, C. S., Mismer, D., Hardy, R. and Rubin, G. M. (1988). Ectopic expression of a minor Drosophila opsin in the major photoreceptor cell class: distinguishing the role of primary receptor and cellular context. Cell 53, 475-482.

Zuker, C. S., Montell, C., Jones, K., Laverty, T. and Rubin, G. M. (1987). A rhodopsin gene expressed in photoreceptor cell R7 of the Drosophila eye: homologies with other signal transducing molecules. J. Neurosci. 7, 1550-1557. 